\title{
OPEN Gestational intermittent hyperoxia rescues murine genetic congenital heart disease in part
}

\author{
Cassandra F. Doll1, ${ }^{1,3}$, Natalia J. Pereira ${ }^{1,3}$, Mustafa S. Hashimi ${ }^{1,3}$, Tabor J. Grindrod ${ }^{1}$, \\ Fariz F. Alkassis ${ }^{1}$, Lawrence X. Cai ${ }^{1}$, Una Milovanovic ${ }^{1}$, Adriana I. Sandino ${ }^{1}$ \& \\ Hideko Kasahara ${ }^{1,2 \bowtie}$
}

Cardiac development is a dynamic process, temporally and spatially. When disturbed, it leads to congenital cardiac anomalies that affect approximately $1 \%$ of live births. Genetic variants in several loci lead to anomalies, with the transcription factor NKX2-5 being one of the largest. However, there are also non-genetic factors that influence cardiac malformations. We examined the hypothesis that hyperoxia may be beneficial and can rescue genetic cardiac anomalies induced by an Nkx2-5 mutation. Intermittent mild hyperoxia $\left(40 \% \mathrm{PO}_{2}\right)$ was applied for $10 \mathrm{~h}$ per day to normal wild-type female mice mated with heterozygous $N k x 2-5$ mutant males from gestational day 8.5 to birth. Hyperoxia therapy reduced excessive trabeculation in Nkx2-5 mutant mice compared to normoxic conditions (ratio of trabecular layer relative to compact layer area, normoxia $1.84 \pm 0.07 \mathrm{vs}$. hyperoxia $1.51 \pm 0.04$ ) and frequency of muscular ventricular septal defects per heart $(1.53 \pm 0.32$ vs. $0.68 \pm 0.15)$; however, the incidence of membranous ventricular septal defects in Nkx2-5 mutant hearts was not changed. Nkx2-5 mutant embryonic hearts showed defective coronary vessel organization, which was improved by intermittent mild hyperoxia. The results of our study showed that mild gestational hyperoxia therapy rescued genetic cardiac malformation induced by $N k \times 2-5$ mutation in part.

Congenital cardiac anomalies are the most prevalent birth defects, affecting approximately $1 \%$ of live births ${ }^{1-3}$. Genetic variants in several loci lead to anomalies ${ }^{4-6}$. Among these, heterozygous homeodomain-containing transcription factor NKX2-5 variants are one of the largest sets (OMIM, NCBI), and currently, nearly 40 variants have been reported in humans ${ }^{7,8}$. The same nucleotide variant can lead to varied types and severity of disease ${ }^{9}$, not only in humans, but also in $N k x 2-5^{+/ R 52 G}$ knock-in mutant mice (hereafter called $N k x 2-5$ mutant) that have the same genetic background through backcrossing ${ }^{8,10,11}$. These observations strongly suggest non-genetic factors have influence on cardiac malformations. In addition, the majority of congenital cardiac anomalies cannot be linked to specific genes ${ }^{9,12}$, suggesting that non-genetic factors alone can lead to cardiac anomalies.

Gestational hypoxia increases the risk of low intrauterine growth, low birth weight, and developing cardiovascular defects. Various conditions such as high-altitude pregnancies, maternal smoking, congestive heart failure, pulmonary diseases, acute/chronic respiratory tract infections, anemia, preeclampsia, and placental insufficiency cause gestational hypoxia ${ }^{13}$. Gestational hypoxia alone can induce cardiac anomalies in experimental animal models ${ }^{14-17}$. Consistently, in our experiments, when wild-type pregnant mice were housed under moderate chronic hypoxia $\left(14 \%\right.$ of $\left.\mathrm{O}_{2}\right)$ conditions from gestational day 10.5 until term, newborn mice showed cardiac anomalies such as excessive trabeculation, ventricular septal defects (VSDs), irregular morphology of interventricular septum as well as atrial septal abnormalities, which overlap with those seen in heterozygous Nkx2-5 mutant mice ${ }^{18}$. In addition, genome-wide transcriptome done by RNA-seq of a 2-day hypoxic exposure on wild-type embryos revealed abnormal transcriptomes in which approximately $60 \%$ share those from $N k \times 2-5$ mutants without hypoxia. Gestational hypoxia reduced the expression of Nkx2-5 proteins in more than one-half, suggesting that abnormal Nkx2-5 function is a common mechanism shared between genetic and gestational hypoxia-induced cardiac anomalies ${ }^{18}$. In heterozygous $N k \times 2-5$ knockout mice, Nkx2-5 expression was also reduced by severe hypoxia at the transcriptional level rather than at the translational level ${ }^{17}$.

${ }^{1}$ Department of Physiology and Functional Genomics, University of Florida College of Medicine, 1600 SW Archer Rd. M543, Gainesville, FL 32610-0274, USA. 'International University of Health and Welfare, School of Medicine, 852 Hatakeda, Narita, Chiba, Japan. ${ }^{3}$ These authors contributed equally: Cassandra F. Doll, Natalia J. Pereira and Mustafa S. Hashimi. ${ }^{\circledR}$ email: hkasahar@ufl.edu 
Advances in clinical care such as surgical interventions have enabled most patients with congenital heart disease to reach adulthood. This prolonged survival, however, has been achieved at a cost, as many patients suffer later complications, of which heart failure and arrhythmias are the most prominent ${ }^{12}$. Alternative treatment aimed at correcting abnormally developing hearts may offer potential in the future ${ }^{8,19}$. Maternal hyperoxygenation has been investigated as a possible therapy for augmenting ventricular growth in fetuses with congenital heart disease, in particular with underdeveloped cardiac chambers (reviewed in Refs. ${ }^{19,20}$ ). The therapeutic potential of hyperoxia also has been tested in rodents with cardiac and noncardiac anomalies, including coarctation of aorta $^{21}$, hypoplastic cardiovascular structure ${ }^{22}$, cleft lip ${ }^{23}$, and phenytoin-induced cleft palate ${ }^{24}$.

High levels of oxygen (i.e., above $80 \% \mathrm{PO}_{2}$ ) has adverse effects such as inducing bronchopulmonary dysplasia, retinopathy, prematurity and perinatal brain injury ${ }^{25-27}$. In this study, we applied mild hyperoxia, and have demonstrated that intermittent hyperoxia $\left(40 \%\right.$ of $\mathrm{O}_{2}, 10 \mathrm{~h}$ per day) starting from gestational day 8.5 partly rescued cardiac anomaly in terms of reducing the frequencies of muscular VSD and ventricular non-compaction with correction of defective coronary vessel organization in Nkx2-5 mutant hearts.

\section{Results}

Micro-computed tomography (CT) imaging is an alternative to serial paraffin-embedded tissue sectioning for detecting cardiac anomalies in postnatal day 1 (P1) mice. Micro-CT has been developing as an alternative method to serial paraffin-embedded tissue sectioning to obtain quantitative imaging of cardiac anomalies. Iodine contrast-enhanced micro-CT scan with $4-\mu \mathrm{m}$ resolution followed by threedimensional (3D) reconstruction was applied to heterozygous P1 Nkx2-5 mutant hearts showing varied cardiac anomalies as reported previously ${ }^{11}$. An example of an Nkx2-5 mutant heart grossly enlarged with expectations of complicated cardiac anomalies was chosen to examine the feasibility and ability of micro-CT imaging (Fig. 1A). Serial paraffin-embedded tissue sectioning of $5 \mu \mathrm{m}$ thickness and micro-CT scanned images side-by-side were comparable and both demonstrated muscular and peri-membranous VSD, double outlet of right ventricle and excessive trabeculation in Nkx2-5 mutant heart (Fig. 1B, tissue section vs. micro-CT). Notably, optical sectioning of micro-CT imaging can be done from any axes, which improves a quality of diagnosis of cardiac anomalies relative to physical histology tissue sectioning (see Supplemental Video S1).

Intermittent mild hyperoxia $\left(40 \% \mathrm{O}_{2}\right)$ for $10 \mathrm{~h}$ per day decreases a number of muscular VSD per heart and the degree of ventricular non-compaction in Nkx2-5 mutant mice. We recently demonstrated that continuous gestational mild hypoxia $\left(14 \% \mathrm{PO}_{2}\right)$ induces cardiac anomalies in wild-type mice, including excessive trabeculation and peri-membranous and muscular VSDs, which partly overlap with those seen in heterozygous $N k \times 2-5$ mutant mice $^{18}$. As opposed to hypoxia, hyperoxia may be effective for rescuing Nkx2-5 mutant mice in part.

In consideration of the toxicity of high levels of oxygen (i.e., above $\left.80 \% \mathrm{PO}_{2}\right)^{25-27}$, and the difficulty in applying continuous $24 \mathrm{~h}$ hyperoxia to pregnant humans with the understanding that this interferes with normal activities of daily living (bathing, meals, and work, etc.), we applied an intermittent mild hyperoxia $\left(40 \% \mathrm{PO}_{2}\right.$, $10 \mathrm{~h}$ per day). Normal wild-type females mated with heterozygous $N k \times 2-5$ mutant males were housed in the hyperoxia chamber during the daytime, which is considered the night in humans, from the gestational day 8.5 to birth (Fig. 2A). Control mice were housed under normoxic conditions. P1 mouse hearts were analyzed using micro-CT two-dimensional (2D) and three-dimensional (3D) imaging.

The most prevalent lesions seen in Nkx2-5 mutant hearts were excessive trabeculations and perimembranous and/or muscular VSDs found in a single or multiple positions ${ }^{11}$. An example of an Nkx2-5 mutant heart showed a perimembranous VSD lacking fibrous continuity between the developing leaflets of the aortic and tricuspid valves, and an additional muscular defect positioned dorsally, being enclosed within the musculature of the septum (Fig. 2B). Mild hyperoxia rescued cardiac anomalies in part by reducing a number of multiple muscular VSDs per heart compared to normoxic conditions ( $1.53 \pm 0.32$ vs. $0.68 \pm 0.15$ ); however, an incidence of perimembranous or muscular VSD was not reduced (Table 1).

Regarding another feature of Nkx2-5 mutant hearts, namely excessive trabeculations, we quantified the total area of the trabecular and compact layers of the walls and interventricular septum areas and compared the relative ratio as described ${ }^{11}$. We first excluded hearts that demonstrated profound anomalies, which obviously changes the ventricular size and morphology, such as double outlets of right ventricle shown in Fig. 1, and then we randomly selected 10 hearts from each group. Although the discrimination between the compact and trabecular layers can be somewhat subjective, we followed consistent criteria throughout the analyses as described in the Materials and Methods. Mild hyperoxia reduced interventricular septum areas relative to the total ventricular area and trabecular layer relative to compact layer area compared to normoxic condition (Fig. 2C,D).

Defective coronary vessel organization in Nkx2-5 mutant embryonic hearts was improved by intermittent mild hyperoxia. The initiation of compact layer thickening (outer layer) and the formation of coronary vasculature occur in an interdependent manner around E11-12 $28-30$. Around E11.5, endothelial cells in the coronary vasculature in the ventricular wall are thought to originate from the sinus venosus localized on the dorsal surface of the myocardium, and migrate between the epicardium and myocardium ${ }^{31-35}$. Because Nkx2-5 mutant hearts show excessive trabeculation, we examined whether coronary vessel organization may be defective in Nkx2-5 mutant mice.

Coronary vessel organization has not been examined previously in $N k \times 2-5$ mutant hearts, thus we first compared it between normoxic wild-type and Nkx2-5 mutant developing hearts using whole-mount immunostaining with the endothelial marker CD31 (Fig. 3A, wild-normoxia vs. mutant-normoxia). Dorsal view of CD31-positive endothelial cell plexus extended toward the apical region in wild-type heart, while it was thinner 


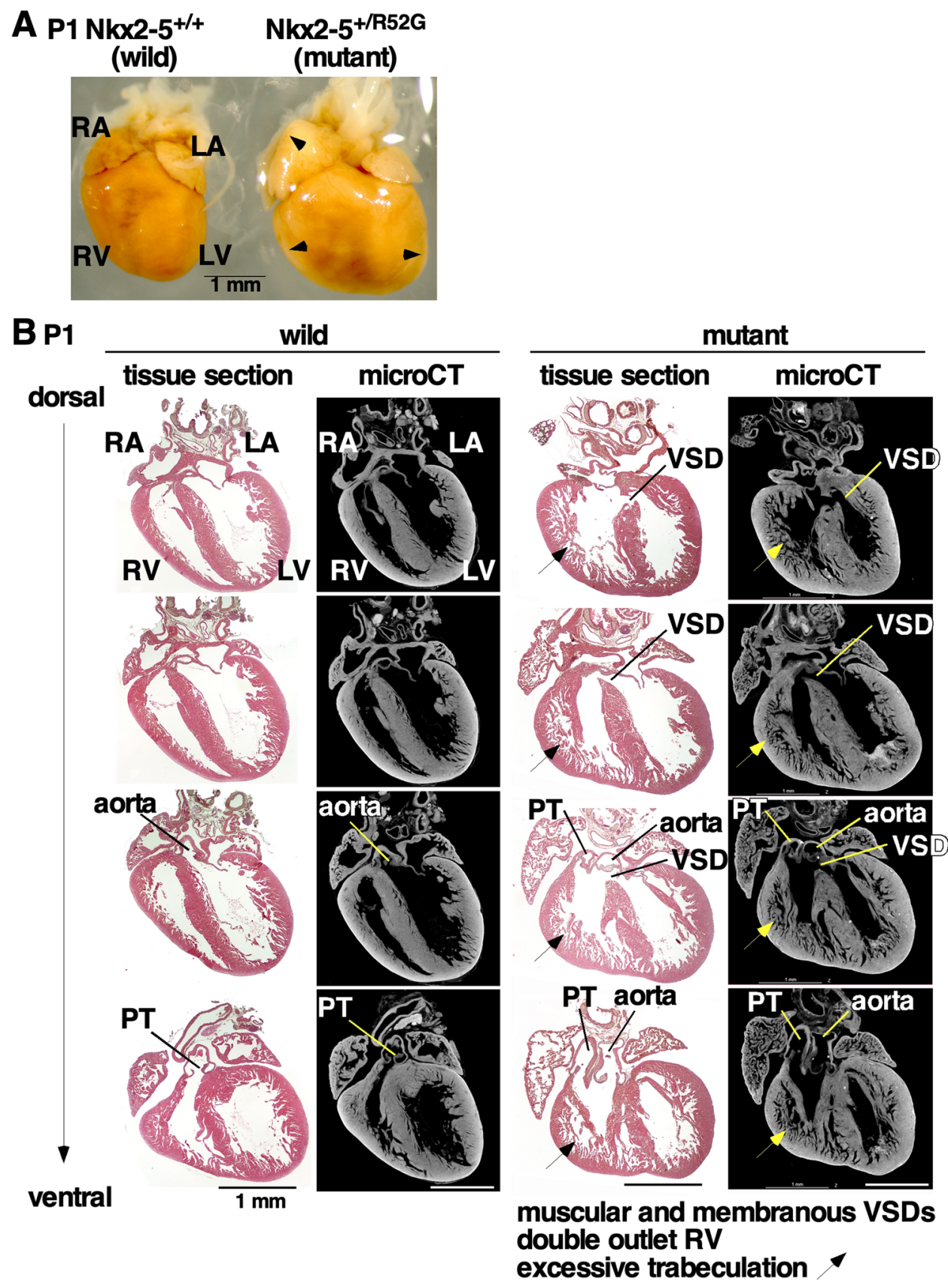

Figure 1. Representative cardiac anomaly in postnatal day $1 \mathrm{Nk} \times 2-5^{+/ R 52 G}$ mouse and control wild-type littermate. Control Nkx2-5/++ (left) vs. $N k \times 2-5^{+/ R 52 G}$ (right): (A) dissected hearts, (B) heart tissue sections and corresponding micro-computed tomography images. Excessive trabeculation in $N k \times 2-5^{+/ R 52 G}$ is indicated by arrows. $L A$ left atrium, $L V$ left ventricle, $P T$ pulmonary trunk, $R A$ right atrium, $R V$ right ventricle, $V S D$ ventricular septal defect.

and less extended in Nkx2-5 mutant heart (Fig. 3A,B, wild-normoxia vs. mutant-normoxia, black arrowheads). Quantitative measurement of the CD31-positive area relative to the total ventricular area showed that it was lower in Nkx2-5 mutant hearts compared to the control wild-type hearts at normoxic conditions with a $P$ value of 0.068 (Fig. 3B, wild-normoxia vs. mutant-normoxia). Of note, we eliminated the peripheral ventricular area for measurement due to high background on the images generated during photo capturing.

Intermittent hyperoxia made CD31-positive coronary vessel organization significantly thicker and more extended relative to normoxic conditions in $N k \times 2-5$ mutant hearts (Fig. 3A,B, mutant-normoxia vs. mutanthyperoxia, black arrowheads). In addition, the compact layer was thicker in E12.5 mutant-hyperoxia hearts relative to the mutant-normoxia hearts with $P$ value below 0.05 in the right ventricle and with $P$ value of 0.054 in the left ventricle (Fig. 3C,D). Overall, there was a correlation between coronary vessel organization and compact layer thickness in E12.5 Nkx2-5 mutant hearts. 
A time course

female (wild) male (mutant)

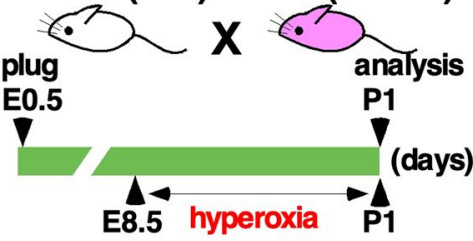

$\mathrm{O}_{2} 40 \%$, intemittent

10 hrs/day for 11 days
B perimembranous muscular
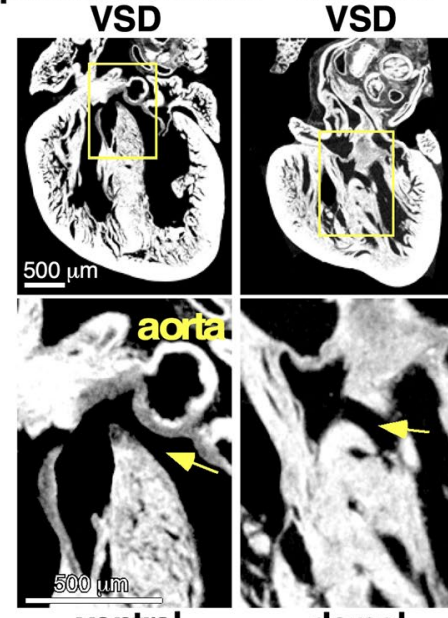

ventral

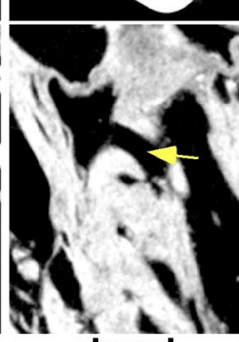

dorsal

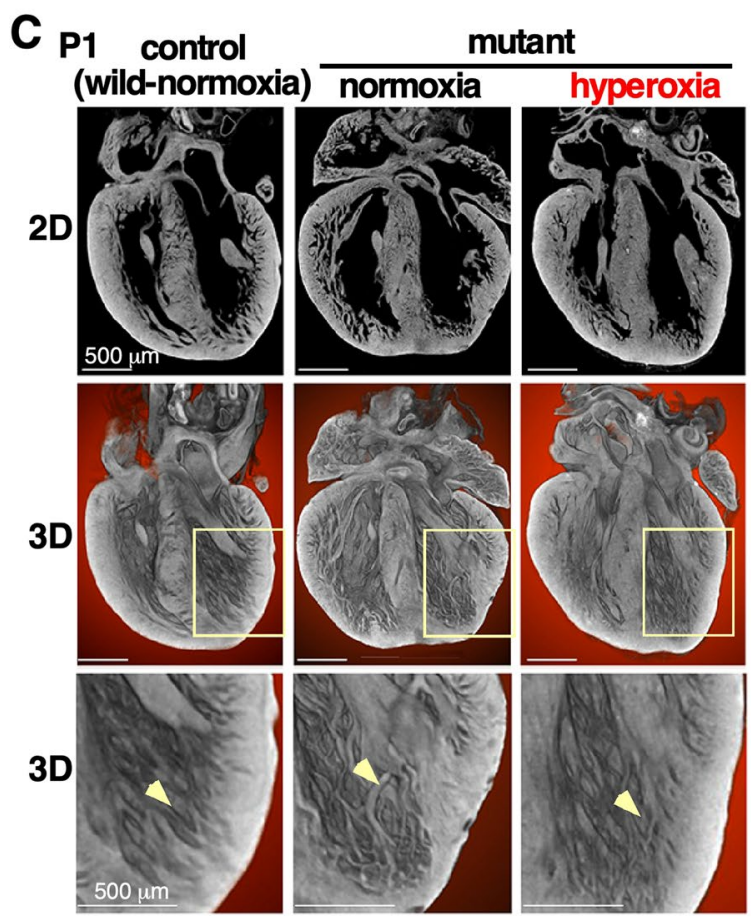

mutant-normoxia $(n=10)$

mutant-hyperoxia $(n=10)$

\section{ratio inter-ventricular septum/total ventricle}

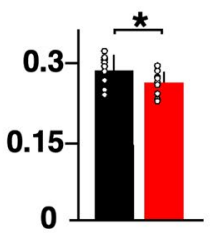

\section{ratio trabecular/ \\ compact}

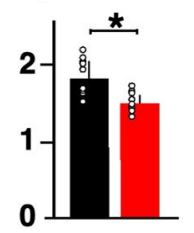

Figure 2. Experimental design of gestational hyperoxia $\left(40 \% \mathrm{O}_{2}\right.$ saturation) and representative images of postnatal day 1 Nkx2-5 mutant hearts using micro-computed tomography (CT). (A) Timeline of experiment. (B) Representative 2D images demonstrating perimembranous and muscular VSD regarding their location (arrows). (C) Representative 2D and 3D micro-CT images showing ventricular trabeculation (arrowheads). (D) Quantification of area size of total ventricle, inter-ventricular septum, trabecular and compact layers. Data are expressed as mean \pm S.D.; individual data are represented as circles. The Shapiro-Wilk normality test confirmed normal distribution of variables. ${ }^{\star} P \leq 0.05$. VSD ventricular septal defect. ImageJ $1.52 \mathrm{~d}$ (http://imagej.nih.gov/ij) was used for analysis.

\begin{tabular}{|l|l|l|l|}
\hline Phenotypes & Mutant-normoxia $(\mathbf{n}=\mathbf{1 9})$ & Mutant-hyperoxia $(\mathbf{n}=\mathbf{2 2})$ & P value \\
\hline Membranous VSD & $6(31.6 \%)$ & $7(31.8 \%)$ & 0.626 \\
\hline Muscular VSD & $13(68.4 \%)$ & $12(54.5 \%)$ & 0.279 \\
\hline Number of muscular VSDs per heart & $1.53 \pm 0.32$ & $0.68 \pm 0.15$ & $0.024^{\star}$ \\
\hline
\end{tabular}

Table 1. Summary of cardiac anomalies of P1 Nkx2-5 mutant mice with or without hyperoxia. The onesided Fisher's exact test was used on a $2 \times 2$ contingency table for calculation of $P$ values for incidence of membranous or muscular VSDs. ${ }^{\star} P \leq 0.05$. 


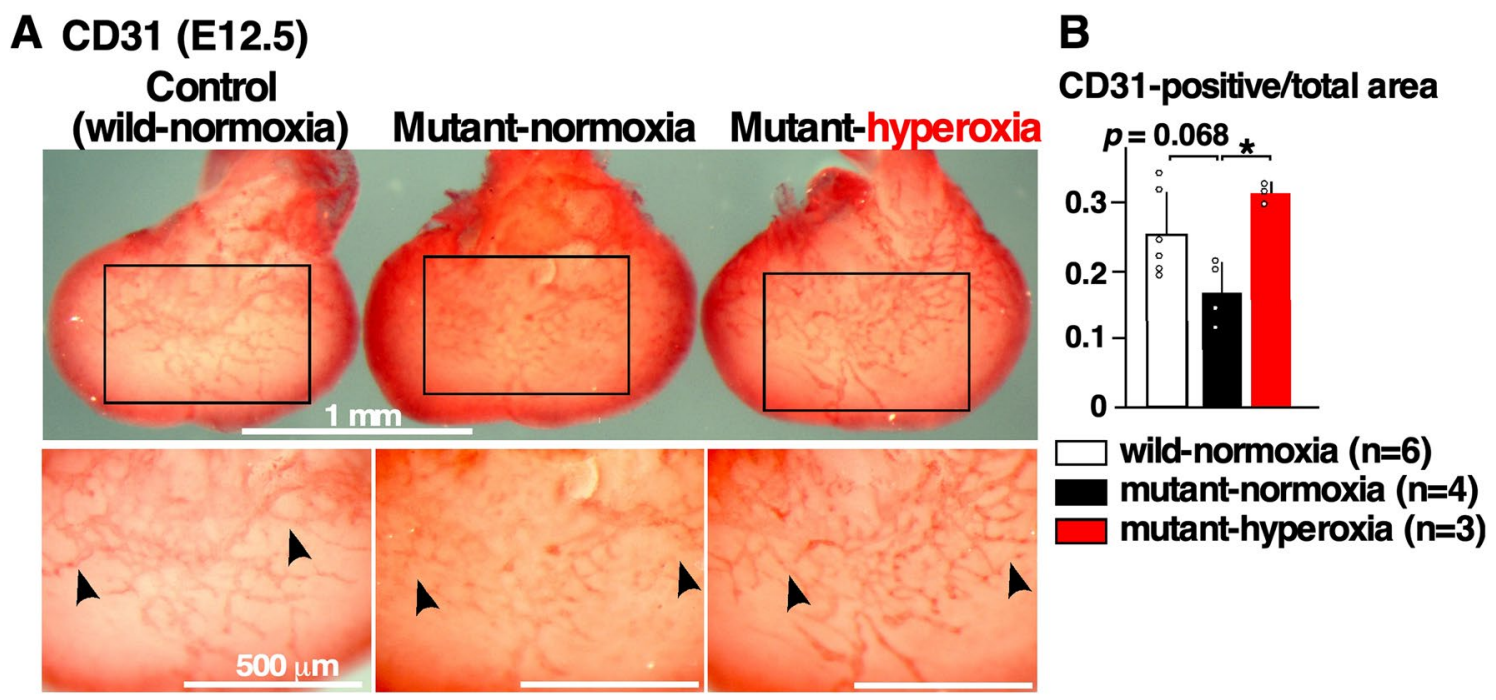

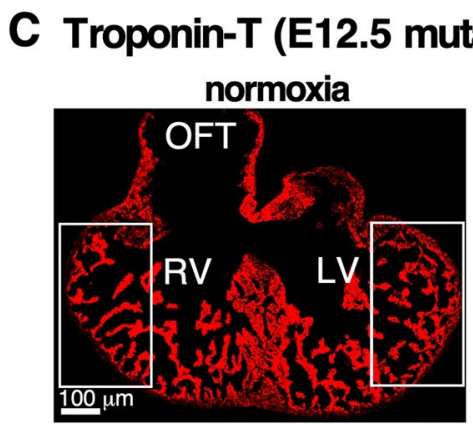

\section{tant )}
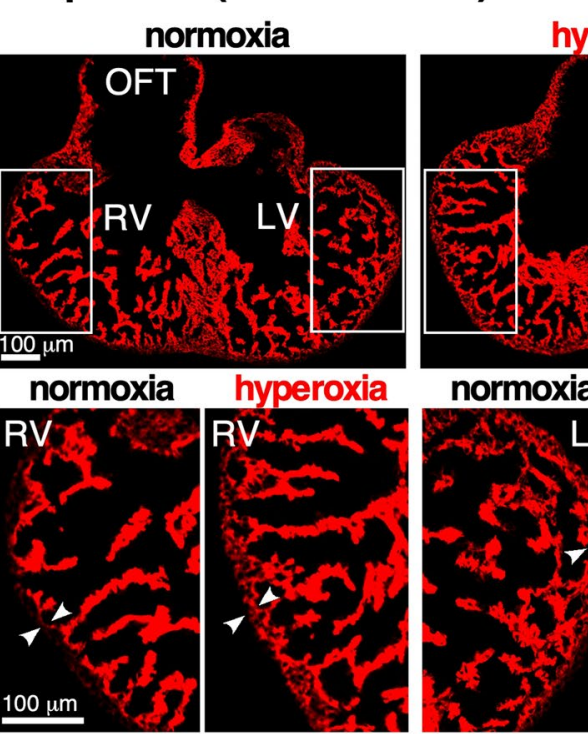

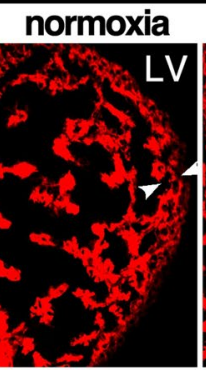

D

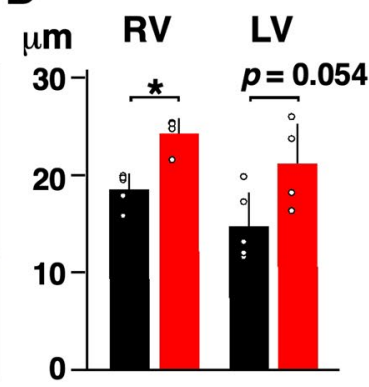

\section{mutant-normoxia $(n=5)$} mutant-hyperoxia $(n=4)$

Figure 3. Defective coronary vessel organization in E12.5 Nkx2-5 mutant embryonic hearts, and the effects of hyperoxia. (A) Dorsal view of whole-mount CD31 immunostaining of ventricles showing endothelial cell plexus organized within the ventricles. CD31 staining was less prominent and extended to the apical area in Nkx2-5 mutant heart at normoxia, but was comparable at hyperoxia to the control wild-type heart (black arrowheads). (B) Quantitative CD31-positive area relative to the entire ventricular area. The number of embryos examined is indicated. The non-parametric Kruskal-Wallis ANOVA with Dunn's test was used for analysis. (C) Representative heart sections stained with troponin-T. Thickness of compact layer is shown in white arrowheads. (D) Quantitative data showing thickness of compact layer in Nkx2-5 mutant hearts at normoxia vs. hyperoxia. The Mann-Whitney $U$ test was used for analysis. Data are expressed as mean \pm S.D.; individual data are represented as circles. ${ }^{\star} P \leq 0.05$. $L V$ left ventricle, $R V$ right ventricle, $O F T$ outflow tract. The number of mice examined is indicated. ImageJ $1.52 \mathrm{~d}$ (http://imagej.nih.gov/ij) was used for analysis.

Increase in Nkx2-5 protein expression by hyperoxia. In our previous studies, we reported that an expression of total Nkx2-5 proteins is the same between $N k \times 2-5$ mutant and wild-type embryos ${ }^{11}$, and Nkx2-5 proteins were reduced by approximately one-half under gestational hypoxia ${ }^{18}$. Mild hyperoxia, in contrast, increased the expression of Nkx2-5 in E12.5 mutant hearts by approximately $2.08 \pm 0.34$-fold relative to normoxic conditions (Fig. 4A,B). However, there was no change in Nkx2-5 mRNA between the two groups similar to the hypoxic conditions (Fig. $4 \mathrm{C}$ ), suggesting that $\mathrm{Nkx} 2-5$ protein expression is regulated post-transcriptionally, consistent to the previous studies ${ }^{18,36}$. 
A E12.5 mutant

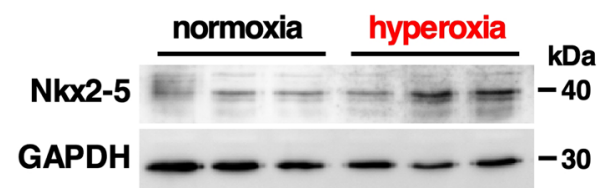

B protein Nkx2-5/GAPDH

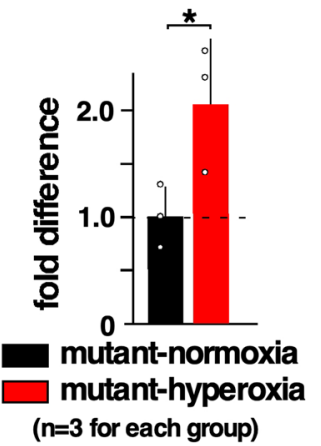

C MRNA

Nkx2-5//actin

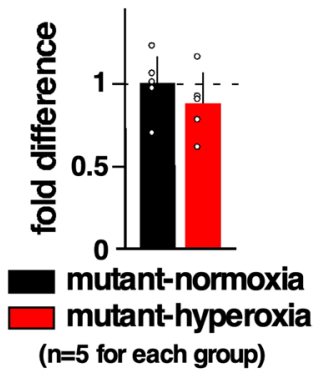

Figure 4. Expression of Nkx2-5 protein in E12.5 Nkx2-5 $5^{+/ R 52 G}$ embryonic hearts with or without hyperoxia. (A) Representative Western blotting demonstrating Nkx2-5 and GAPDH proteins (see Supplemental Fig. S1). (B) Quantitative data for Nkx2-5 protein expression relative to GAPDH. (C) Real-time reverse transcriptase PCR of Nkx2-5 mRNA relative to $B$-actin. The value in the Nkx2-5 mutant-normoxia was defined as 1 . The MannWhitney U-test was used for analysis. Data are expressed as mean \pm S.D.; individual data are represented as circles. ${ }^{\star} P \leq 0.05$. ImageJ $1.52 \mathrm{~d}$ (http://imagej.nih.gov/ij) was used for analysis.

\section{Discussion}

Hyperoxygenation treatment for pregnant women with a fetal diagnosis of congenital heart disease has been studied and there has been some success in improving cardiac growth (reviewed in Co-VU et al. ${ }^{20}$ ). We applied intermittent mild hyperoxia $\left(40 \% \mathrm{O}_{2}, 10 \mathrm{~h}\right.$ per day) to wild-type pregnant mice with the expectation of having fetuses with genetic congenital heart disease induced by an Nkx2-5 mutation from gestational day 8.5 to birth. The frequency of muscular VSD found in the heart and excessive trabeculation were significantly reduced by gestational hyperoxia compared to normoxic conditions. In addition, defective coronary vessel organization in Nkx2-5 mutant embryonic hearts was improved by intermittent mild hyperoxia. These data suggest that gestational hyperoxia rescues genetic cardiac anomalies to some degree with statistical significance, but not completely. Non-genetic factors that aggravate or induce cardiac anomalies were relatively well documented ${ }^{9,12}$, but factors that reduce or cure such anomalies are not well studied.

According to a recent review study by $\mathrm{Co}-\mathrm{Vu}$ et al., maternal hyperoxygenation therapy is practical, safe and effective in fetuses with congenital heart disease, especially in the growth of hypoplastic left heart structures ${ }^{20}$. In these studies, therapies were applied during the second and third trimester, in contrast to the current hyperoxia therapy, which was applied beginning from gestational day 8.5 , which translates to human gestation at 2.5-3 weeks. Among nine studies reviewed, only one used a chronic hyperoxygenation therapy protocol with 8-9 L/min 100\% oxygen for over $8 \mathrm{~h}$ per day (excluding time for bathing, meals, and work) by wearing a non-rebreather mask beginning from an average 29.6 weeks to delivery ${ }^{37}$. The treatment appeared feasible and improved aortic and mitral annular growth relative to the control group without statistical significance due to the low number of participants ( $n=9$ each). Regarding rescue of left ventricular hypoplasia by maternal hyperoxygenation, the timing of hyperoxia therapy is important; it is effective between 31 and 36 weeks of gestation, but not between 20 and 26 weeks ${ }^{38}$. Clinical trials with more participants will be necessary to demonstrate statistical significance for the hyperoxygenation treatment.

Human conception occurs 2 weeks before an expected period, thus appropriate pregnancy tests should be positive on the first day of a missed period ${ }^{39,40}$. In the clinical setting, it is possible for parents to know their genetic abnormalities in the Nkx2-5 gene through genetic counseling. Therefore, the application of intermittent hyperoxia therapy beginning from human gestation 2.5-3 weeks might be feasible in the case that a woman actively trying to conceive starts the therapy when she misses an expected period and confirms her human chorionic gonadotropin-positive pregnancy test.

After testing feasibility and resolution of micro-CT imaging that shows comparability to traditional serial paraffin-tissue sectioning, we used micro-CT scanning with 4 - $\mu \mathrm{m}$ resolution to assess cardiac anomalies in P1 mice. Scanning time for $4-\mu \mathrm{m}$ resolution was around $30 \mathrm{~min}$ for one P1 heart, and it could be shorter to achieve lower resolutions, such as at $25-50 \mu \mathrm{m}^{41}$. An additional soft-tissue contrast agents (Lugol's solution) was used in this study to enhance contrast and resolution ${ }^{41}$. We not only reduced the time and effort to examine cardiac anomalies, but analyzed the images interpolated into a 3D reconstruction from multiple directions as shown in the Supplemental Video S1. 3D reconstructed imaging from serial paraffin-embedded tissue sectioning is achievable; however it requires extensive time and technical skills ${ }^{11,42}$. The inclusion of quantitative image analyses, such as that using Osirix software ${ }^{43}$, will be considered in the future.

In Nkx2-5 mutant embryonic hearts, we found defective coronary vessel organization compared to the wildtype, for the first time to our knowledge, which was improved by gestational hyperoxia at E12.5. Although angiogenesis is generally thought to be induced by hypoxia ${ }^{44-46}$, coronary vessel organization in embryonic hearts was opposingly made defective by gestational hypoxia ${ }^{47}$. In addition, gestational hyperoxia improved coronary vessel organization in $N k \times 2-5$ mutant hearts. We speculate that improved coronary vessel organization helped 
compact layer thickening and modified trabecular layer structure in $N k x 2-5$ mutant hearts. Considering that the ventricular septum is itself formed by compaction of the trabecular layer ${ }^{29}$, this offers an obvious correlation between the noncompaction and the multiple muscular defects in $N k x 2-5$ mutant hearts ${ }^{11}$, both of which were improved by hyperoxia treatment.

Gestational hyperoxia was applied at a single concentration $\left(40 \% \mathrm{O}_{2}\right)$ beginning on gestational day 8.5. We could not measure blood oxygen levels in pregnant mothers or fetuses housed in the hyperoxia chamber, or test the different oxygen concentrations. The timing of hyperoxia application was tested in pilot studies beginning on E8.5 $(n=22)$ or E10.5 $(n=7)$, and E8.5 appears more effective for ventricular compaction from the micro-CT imaging without statistical analyses. Different oxygen concentrations as well as the timing of exposure need to be investigated in the future. To quantitate the level of Nkx2-5 protein, we loaded the same amount of protein and normalized it with GAPDH expression to eliminate loading errors using standard methodology. In adult mouse hearts, however, the expression of house-keeping proteins, such as GAPDH, tubulin, and actin is altered by 28 -day hyperoxia $\left(30 \% \mathrm{O}_{2}\right)$ exposure ${ }^{48}$. This must be considered when analyzing a level of protein expression relative to GAPDH.

There are several additional limitations in this study. First, the mechanism by which mild hyperoxia improves cardiac anomalies in Nkx2-5 mutants is not clear. Second, the present study lacked information regarding the details of defective coronary vessel organization in Nkx2-5 mutants. Those include stage-dependent changes, underlying mechanisms regarding how a mutation in the $N k \times 2-5$ gene affects vascular developments despite Nkx2-5 not being expressed in endothelial cells or smooth muscle cells ${ }^{49}$, and how it is improved by hyperoxia. Third, the direct correlation between defective coronary vessel organization and cardiac anomalies including ventricular non-compaction in Nkx2-5 mutants is not clear.

In summary, we report a potential therapy using mild gestational hyperoxia for genetic cardiac malformation induced by an Nkx2-5 mutation.

\section{Materials and methods}

The study was carried out in accordance with relevant guidelines and regulations, including compliance with the ARRIVE guidelines.

Animal models with hyperoxia. The animal models were reported in our previous studies ${ }^{11,18}$. In short, wild-type $129 / \mathrm{Sv}$ female mice were placed in a hyperoxic chamber (COY Lab Products, Grass Lake, MI) that was connected to nitrogen and oxygen gas. The oxygen content was gradually increased from 20.9 to $40 \%$ over $15 \mathrm{~min}$ and the carbon dioxide was absorbed by Carbolime (AliMed, Inc., Dedham, MA). The cages were kept for $10 \mathrm{~h}$, and returned to the normoxic condition for $14 \mathrm{~h}$ daily. On P1, hearts were examined for cardiac anomalies. At gestational day 12.5, mice were sacrificed immediately after moving them from the hyperoxic chamber to maintain hyperoxic conditions. All animal experiments were performed with approval from the University of Florida Institutional Animal Care and Use Committee.

Micro-CT imaging. To stop heartbeat at diastole, hearts were soaked in $10 \% \mathrm{KCl} / \mathrm{PBS}$ immediately after dissection, and then fixed with $4 \%$ paraformaldehyde overnight at $4{ }^{\circ} \mathrm{C}$, washed with PBS and kept in $70 \%$ ethanol. Two days before imaging, hearts were rehydrated with PBS, stained with iodine solution $\left(2.5 \% \mathrm{KI}\right.$ and $1.3 \% \mathrm{I}^{2}$ in $\mathrm{ddH}_{2} \mathrm{O}$ ) overnight, and then washed with PBS following the protocol ${ }^{41}$. Images were obtained using GE Phoenix V|TOME|X M 240 Micro-CT (GE Sensing and Inspection Technologies GmbH, Wunstorf, Germany, Nanoscale Institute, University of Florida, Gainesville, FL). By stacking cross-sectionally sliced images, 3D X-ray images of neonatal mouse hearts were generated and analyzed by the Dragonfly program (Object Research Systems, Quebec, Canada) and ImageJ.

Digitalized images were used for counting the number of VSD, as well as for measuring the total area, the interventricular septum, and trabecular and compact layers. The number of VSD per heart were examined in whole hearts from multiple directions. A longitudinal section showing the largest left and right ventricular cavity was selected from the 3D images for measuring the total area, the interventricular septum, and trabecular and compact layers. The border between trabecular and compact layers was somewhat difficult to determine; we used the morphological border with the deepest endomyocardial recesses at the diastolic phase ${ }^{50}$, as described in our previous studies ${ }^{11,18,42,51}$. Just as importantly, some of our previous studies include a genetic marker for trabecular layers that we analyzed using atrial natriuretic factor-lacZ transgenic mice ${ }^{51}$.

Histological analysis and Western blotting. Serial paraffin-embedded tissue sectioning of $5 \mu \mathrm{m}$ thickness was used for histological analysis and immunostaining as described ${ }^{11}$. Immunostaining and Western blotting were performed with the following primary antibodies: CD31 (CM303, Biocare Medical, Pacheco, CA), Nkx2-549, and troponin T (T6277, Sigma, St. Louis, MO). For whole-mount CD31 staining, after incubation with anti-CD31 antibody at $4{ }^{\circ} \mathrm{C}$ overnight, Rat-on-Mouse AP-Polymer (RT518, Biocare Medical, Pacheco, CA) was applied and visualized with Liquid Permanent Red (K0640, Agilent Dako, Santa Clara, CA). Whole-mount images were obtained with SMZ800 (Nikon, Melville, NY) attached to a CCD camera, and fluorescent microscopic images were obtained using Axiovert200M (ZEISS, Oberkochen, Germany). Digitalized images from tissue-staining were used for measurement with Image J software as described previously ${ }^{42,47}$.

Real-time reverse transcriptase (RT)-PCR. RT-PCR was performed using inventoried TaqMan gene expression assays, Nkx2-5 Mm00657783_m1 (Applied Biosystems, Foster City, CA), and normalized to B-actin expression (no. 4352933E). Duplicate experiments were averaged. 
Statistical analysis. Results were analyzed by SPSS (version 26). Data presented are expressed as mean values plus or minus the standard deviation (S.D.). The Shapiro-Wilk normality test was used to assess normal distribution. The Mann-Whitney U test was used to compare continuous variables without normal distribution or when the sample number was small. The independent T-test was used to compare continuous variables with normal distribution. The non-parametric Kruskal-Wallis ANOVA with Dunn's test was used for comparison of three groups in Fig. 3B. The one-sided Fisher's exact test was used for Table 1. $P$ values $\leq 0.05$ were considered significant.

Received: 23 January 2020; Accepted: 22 February 2021

Published online: 23 March 2021

\section{References}

1. Hoffman, J. I., Kaplan, S. \& Liberthson, R. R. Prevalence of congenital heart disease. Am. Heart J. 147, 425-439 (2004).

2. Capozzi, G. et al. Congenital heart disease in live-born children: Incidence, distribution, and yearly changes in the Campania Region. J. Cardiovasc. Med. (Hagerstown) 9, 368-374 (2008).

3. Dolk, H., Loane, M. \& Garne, E. Congenital heart defects in Europe: Prevalence and perinatal mortality, 2000 to 2005. Circulation 123, 841-849 (2011)

4. Muntean, I., Toganel, R. \& Benedek, T. Genetics of congenital heart disease: Past and present. Biochem. Genet. 55, 105-123 (2017).

5. Blue, G. M. et al. Advances in the genetics of congenital heart disease: A clinician's guide. J. Am. Coll. Cardiol. 69, 859-870 (2017).

6. Zaidi, S. \& Brueckner, M. Genetics and genomics of congenital heart disease. Circ. Res. 120, 923-940 (2017).

7. Kasahara, H. \& Benson, D.W. Biochemical analyses of eight NKX2.5 homeodomain missense mutations causing atrioventricular block and cardiac anomalies. Cardiovasc. Res. 64, 40-51 (2004).

8. Benson, D. W. Genetic origins of pediatric heart disease. Pediatr. Cardiol. 31, 422-429 (2010).

9. Postma, A. V., Bezzina, C. R. \& Christoffels, V. M. Genetics of congenital heart disease: The contribution of the noncoding regulatory genome. J. Hum. Genet. 61, 13-19 (2016).

10. Benson, D.W., et al. Mutations in the cardiac transcription factor NKX2.5 affect diverse cardiac developmental pathways. J. Clin. Invest. 104, 1567-1573 (1999).

11. Ashraf, H. et al. A mouse model of human congenital heart disease: high incidence of diverse cardiac anomalies and ventricular noncompaction produced by heterozygous nkx2-5 homeodomain missense mutation. Circ. Cardiovasc. Genet. 7, 423-433 (2014).

12. van der Bom, T. et al. The changing epidemiology of congenital heart disease. Nat. Rev. Cardiol. 8, 50-60 (2011).

13. Jang, E. A., Longo, L. D. \& Goyal, R. Antenatal maternal hypoxia: Criterion for fetal growth restriction in rodents. Front. Physiol. 6, 176 (2015).

14. Kenchegowda, D. et al. Vulnerability of the developing heart to oxygen deprivation as a cause of congenital heart defects. J. Am. Heart Assoc. 3, e000841 (2014).

15. Shi, H. et al. Gestational stress induces the unfolded protein response, resulting in heart defects. Development 143, 2561-2572 (2016).

16. Yuan, X. et al. Disruption of spatiotemporal hypoxic signaling causes congenital heart disease in mice. J. Clin. Invest. 127, 2235-2248 (2017).

17. Moreau, J.L.M., et al. Gene-environment interaction impacts on heart development and embryo survival. Development 146(2019).

18. Moumne, O. et al. Mechanism sharing between genetic and gestational hypoxia-induced cardiac anomalies. Front. Cardiovasc. Med. 5, 100 (2018).

19. Roybal, J. L., Santore, M. T. \& Flake, A. W. Stem cell and genetic therapies for the fetus. Semin. Fetal Neonatal. Med. 15, 46-51 (2010).

20. Co-Vu, J., Lopez-Colon, D., Vyas, H. V., Weiner, N. \& DeGroff, C. Maternal hyperoxygenation: A potential therapy for congenital heart disease in the fetuses? A systematic review of the current literature. Echocardiography 34, 1822-1833 (2017).

21. Zeng, S. et al. Sustained maternal hyperoxygenation improves aortic arch dimensions in fetuses with coarctation. Sci. Rep. 6, 39304 (2016).

22. Kohl, T. Chronic intermittent materno-fetal hyperoxygenation in late gestation may improve on hypoplastic cardiovascular structures associated with cardiac malformations in human fetuses. Pediatr. Cardiol. 31, 250-263 (2010).

23. Millicovsky, G. \& Johnston, M. C. Hyperoxia and hypoxia in pregnancy: Simple experimental manipulation alters the incidence of cleft lip and palate in CL/Fr mice. Proc. Natl. Acad. Sci. USA 78, 5722-5723 (1981).

24. Millicovsky, G. \& Johnston, M. C. Maternal hyperoxia greatly reduces the incidence of phenytoin-induced cleft lip and palate in A/J mice. Science 212, 671-672 (1981).

25. Altemeier, W. A. \& Sinclair, S. E. Hyperoxia in the intensive care unit: why more is not always better. Curr. Opin. Crit. Care 13, 73-78 (2007).

26. Perrone, S., Bracciali, C., Di Virgilio, N. \& Buonocore, G. Oxygen use in neonatal care: A two-edged sword. Front. Pediatr. 4, 143 (2016).

27. Reich, B., Hoeber, D., Bendix, I. \& Felderhoff-Mueser, U. Hyperoxia and the immature brain. Dev. Neurosci. 38, 311-330 (2016).

28. Anderson, R. H. et al. Key questions relating to left ventricular noncompaction cardiomyopathy: Is the emperor still wearing any clothes?. Can. J. Cardiol. 33, 747-757 (2017).

29. Sedmera, D., Pexieder, T., Vuillemin, M., Thompson, R. P. \& Anderson, R. H. Developmental patterning of the myocardium. Anat. Rec. 258, 319-337 (2000).

30. Bhattacharya, S., Macdonald, S. T. \& Farthing, C. R. Molecular mechanisms controlling the coupled development of myocardium and coronary vasculature. Clin. Sci. (Lond) 111, 35-46 (2006).

31. Red-Horse, K., Ueno, H., Weissman, I. L. \& Krasnow, M. A. Coronary arteries form by developmental reprogramming of venous cells. Nature 464, 549-553 (2010).

32. Wu, B. et al. Endocardial cells form the coronary arteries by angiogenesis through myocardial-endocardial VEGF signaling. Cell 151, 1083-1096 (2012).

33. Caballero, S., Correa-Cardenas, C. A. \& Trujillo, F. Population structure and genetic diversity of the endangered South American Giant Otter (Pteronura brasiliensis) from the Orinoco Basin in Colombia: Management implications and application to current conservation programs. J. Hered. 106(Suppl 1), 469-477 (2015).

34. Sharma, B., et al. Alternative progenitor cells compensate to rebuild the coronary vasculature in Elabela- and Apj-deficient hearts. Dev. Cell 42, 655-666 e653 (2017).

35. Hu, S. \& Kurpios, N. A. Coronary arteries shake up developmental dogma. Dev. Cell 47, 680-681 (2018).

36. Nie, J. et al. Post-transcriptional regulation of Nkx2-5 by RHAU in heart development. Cell Rep. 13, 723-732 (2015). 
37. Lara, D. A. et al. Pilot study of chronic maternal hyperoxygenation and effect on aortic and mitral valve annular dimensions in fetuses with left heart hypoplasia. Ultrasound Obstet. Gynecol. 48, 365-372 (2016).

38. Rasanen, J. et al. Reactivity of the human fetal pulmonary circulation to maternal hyperoxygenation increases during the second half of pregnancy: A randomized study. Circulation 97, 257-262 (1998).

39. Chard, T. Pregnancy tests: a review. Hum Reprod 7, 701-710 (1992).

40. Morse, J. E. et al. Evidence-based pregnancy testing in clinical trials: Recommendations from a multi-stakeholder development process. PLoS ONE 13, e0202474 (2018).

41. Gregg, C. L., Recknagel, A. K. \& Butcher, J. T. Micro/nano-computed tomography technology for quantitative dynamic, multi-scale imaging of morphogenesis. Methods Mol. Biol. 1189, 47-61 (2015).

42. Terada, R. et al. Ablation of Nkx2-5 at mid-embryonic stage results in premature lethality and cardiac malformation. Cardiovasc. Res. 91, 289-299 (2011).

43. Captur, G. et al. Morphogenesis of myocardial trabeculae in the mouse embryo. J. Anat. 229, 314-325 (2016).

44. Forsythe, J. A. et al. Activation of vascular endothelial growth factor gene transcription by hypoxia-inducible factor 1. Mol. Cell Biol. 16, 4604-4613 (1996).

45. Damert, A., Ikeda, E. \& Risau, W. Activator-protein-1 binding potentiates the hypoxia-induciblefactor-1-mediated hypoxia-induced transcriptional activation of vascular-endothelial growth factor expression in C6 glioma cells. Biochem. J. 327(Pt 2), 419-423 (1997).

46. Arcondeguy, T., Lacazette, E., Millevoi, S., Prats, H. \& Touriol, C. VEGF-A mRNA processing, stability and translation: A paradigm for intricate regulation of gene expression at the post-transcriptional level. Nucleic Acids Res. 41, 7997-8010 (2013).

47. Cai, L.X., Alkassis, F.F. \& Kasahara, H. Defective coronary vessel organization and reduction of VEGF-A in mouse embryonic hearts with gestational mild hypoxia. Dev. Dyn. (2020).

48. Gyongyosi, A. et al. The impact of moderate chronic hypoxia and hyperoxia on the level of apoptotic and autophagic proteins in myocardial tissue. Oxid. Med. Cell Longev. 2018, 5786742 (2018).

49. Kasahara, H., Bartunkova, S., Schinke, M., Tanaka, M. \& Izumo, S. Cardiac and extracardiac expression of Csx/Nkx25 homeodomain protein. Circ Res. 82, 936-946 (1998).

50. Paterick, T. E. et al. Left ventricular noncompaction: a 25-year odyssey. J. Am. Soc. Echocardiogr. 25, 363-375 (2012).

51. Warren, S. A. et al. Differential role of Nkx2-5 in activation of the atrial natriuretic factor gene in the developing versus failing heart. Mol. Cell Biol. 31, 4633-4645 (2011).

\section{Acknowledgements}

This work was supported by the National Institutes of Health (Grant no. 1R21 HD090608) and the University Florida Opportunity Fund (to H.K.). We are grateful to the UF Nanoscience Institute for Medical \&Engineering Technology for nano/micro-CT imaging, and to E. Chan and C. Astrom for valuable suggestions and technical support.

\section{Author contributions}

Experiments were designed and performed by C.F.D., N.J.P., M.S.H., T.J.G., F.F.A., L.X.C., U.M., A.I.S., and H.K. The manuscript was prepared by L.X.C. and H.K. All authors read and approved the final manuscript.

\section{Competing interests}

Dr. Kasahara's work has been funded by the National Institutes of Health and the University of Florida. Other authors declare no competing interests.

\section{Additional information}

Supplementary Information The online version contains supplementary material available at https://doi.org/ 10.1038/s41598-021-85569-9.

Correspondence and requests for materials should be addressed to H.K.

Reprints and permissions information is available at www.nature.com/reprints.

Publisher's note Springer Nature remains neutral with regard to jurisdictional claims in published maps and institutional affiliations.

Open Access This article is licensed under a Creative Commons Attribution 4.0 International License, which permits use, sharing, adaptation, distribution and reproduction in any medium or format, as long as you give appropriate credit to the original author(s) and the source, provide a link to the Creative Commons licence, and indicate if changes were made. The images or other third party material in this article are included in the article's Creative Commons licence, unless indicated otherwise in a credit line to the material. If material is not included in the article's Creative Commons licence and your intended use is not permitted by statutory regulation or exceeds the permitted use, you will need to obtain permission directly from the copyright holder. To view a copy of this licence, visit http://creativecommons.org/licenses/by/4.0/.

(c) The Author(s) 2021 